\title{
Early Perfusion MRI Predicts Survival Outcome in Patients with Recurrent Glioblastoma Treated with Bevacizumab and Carboplatin
}

Iwan E. Bennett, Kathryn M. Field, Christopher M. Hovens, Bradford A. Moffat, Mark A. Rosenthal, Katharine Drummond, Andrew H. Kaye, and Andrew P. Morokoff

Department of Surgery, The University of Melbourne, $6^{\text {th }}$ Floor, Clinical Sciences Building, The Royal Melbourne Hospital, Royal Parade, Parkville, Victoria, Australia 3050 (I.B., C.H., K.D., A.K., A.M.); Department of Radiology, The University of Melbourne, $1^{\text {st }}$ Floor, The Royal Melbourne Hospital, 300 Grattan St, Parkville, Victoria, Australia 3050 (B.M.); Departments of Medical Oncology (K.F., M.R.) and Neurosurgery (K.D., A.K., A.M.), The Royal Melbourne Hospital, 300 Grattan St, Parkville, Victoria, Australia 3050.

Corresponding Author: Dr. Andrew Morokoff, Department of Surgery, The University of Melbourne, 6th Floor, Clinical Sciences Building, The Royal Melbourne Hospital, Royal Parade, Parkville, Victoria, Australia 3050. Phone +61-3-9035-8586; Fax +61-3-9347-6488; E-mail: morokoff@unimelb.edu.au

Funding: The CABARET trial was conducted by The Cooperative Trials Group for NeuroOncology (COGNO), and was funded in part by Roche. This analysis was supported in part by funding received from The Brain Foundation, the Neurosurgical Society of Australasia, the Cure for Life Foundation, and the Royal Australasian College of Surgeons. 
Acknowledgments: The authors wish to thank Luisa Barassi, Linda Garrett, Simon Salinas and Chris Steward for their assistance in this project.

Conflicts of Interest: The CABARET trial was funded in part by Roche. No other potential conflicts of interest regarding the submission of this manuscript have been identified by any of the authors.

\footnotetext{
Abstract

Background: Bevacizumab (B), an anti-angiogenic agent, is FDA-approved for use in patients with recurrent glioblastoma multiforme (rGBM). The radiologic evaluation of tumor response to $\mathrm{B}$ is complex and there is no validated method of monitoring tumor vascularity during therapy.

Methods: We evaluated perfusion-weighted MR imaging (PWI) in our cohort of patients enrolled in the CABARET trial, which examined the effectiveness of B with or without carboplatin in patients with rGBM. Pre-treatment and early follow-up (4- and 8-week) PWI were used to calculate relative cerebral blood volume (rCBV) histogram statistics of the contrast-enhancing and FLAIR hyperintense tumor volumes. A novel rCBV measurement (load) was developed to estimate the total volume of perfused tumor blood vessels. Changes in all rCBV measures were examined for correlations with progression-free (PFS) and overall survival (OS).

Results: All of our 15 patients enrolled in the CABARET trial were included. Median PFS and OS were 23 and 45 weeks respectively. Kaplan Meier analysis of pre-treatment PWI revealed an 18 week reduction in median OS in patients with high tumor $\mathrm{rCBV}(\mathrm{p}=0.031)$. Changes in rCBV measures, especially load, correlated significantly with PFS and OS at both follow-up time-points. Patients with the greatest reduction in $\mathrm{rCBV}_{\text {load }}$ by 8 -weeks of therapy had a significantly increased median OS (30 weeks; $\mathrm{p}=0.013$ ).

Conclusions: PWI may be of significant clinical utility in managing patients with rGBM, particularly those treated with anti-angiogenic agents such as B. These findings need to be confirmed prospectively in larger studies.

Key words: bevacizumab, glioblastoma, DSC, MRI, rCBV, perfusion.
} 


\section{Introduction}

Bevacizumab is a humanized murine monoclonal antibody targeting vascular endothelial growth factor-A, and is approved for the treatment of recurrent glioblastoma (rGBM) based on initial reported response rates of $46 \%$ [1]. Subsequent phase II studies have further demonstrated its efficacy in salvage therapy [2]. However, the progression-free survival (PFS) benefits of bevacizumab have not yet translated into improved overall survival (OS) in either the de novo or recurrent disease settings.

One concern is that MRI based assessment of bevacizumab response may be inaccurate and misleading. Pseudo-response is well described when tumors are assessed by conventional MacDonald criteria due to decreasing tumor vessel permeability causing decreased contrast enhancement [2]. The RANO criteria were introduced to specifically account for this issue [3]. Newer imaging modalities not dependent on contrast-enhancement are also being investigated for determining outcome measures in GBM [4].

Perfusion MRI, or perfusion-weighted imaging (PWI), rapidly obtains images to serially measure the movement of a tracer agent within a tissue of interest to give estimates of its haemodynamic properties. Reductions in PWI parameters such as permeability coefficients (e.g. $\mathrm{K}^{\text {trans }}$ ) and cerebral blood volume (CBV) have been demonstrated with bevacizumab administration in both pre-clinical studies [5-7] and phase II clinical trials [8]. The ability of PWI to predict patient outcome remains uncertain, with mixed results being reported from the small number of studies investigating this relationship [9-11]. 
This study retrospectively examined the prognostic value of PWI specifically in patients with rGBM treated with bevacizumab. In particular, the analysis included pre-treatment scans and an "early" time-point scan undertaken after only four weeks of treatment.

\section{Materials and Methods}

\section{Patient Selection and Follow-up}

Study participants were those patients recruited from The Royal Melbourne and Melbourne Private Hospitals for the CABARET randomized phase II clinical trial. Inclusion and exclusion criteria for the CABARET trial are listed in the supplementary material. Briefly, patients eligible for inclusion were those over 18 years old with a tissue diagnosis of GBM and disease recurrence on post-contrast MRI or confirmed histologically. Previous treatment with temozolomide and radiotherapy was mandatory. Patients were excluded if there was any evidence of recent intracerebral hemorrhage or an inability to undergo MRI. The study was conducted with institutional Human Research Ethics Committee approval.

Patient age, gender, and presenting functional status (KPS, Karnofsky Performance Status) were recorded at enrolment. Patients were followed prospectively to determine PFS and OS. Progression-free survival was defined as the time from randomization to clinical or radiographic progression. Overall survival was determined by the time from randomization to death. 


\section{CABARET Trial Protocol}

The CABARET trial is a randomized phase II multi-center study in patients with rGBM consisting of two parts. Part 1 randomized patients to bevacizumab alone or bevacizumab plus carboplatin. At disease progression, Part 2 randomized patients suitable for further therapy to either continuation of bevacizumab or discontinuation. Part 2 allowed patients to be treated with physicians' choice chemotherapy (carboplatin, temozolomide or etoposide).

\section{MR Imaging}

MRI findings from pre-treatment scans, as well as the initial two follow-up scans during Part 1 of the trial were assessed for their ability to predict outcome. The first two follow-up scans were undertaken at 4- and 8-weeks following randomization as per trial protocol. While 8-week follow-up imaging is not unusual in studies of this kind as well as in clinical practice, the 4-week follow-up MRI represented a deliberate early time-point for radiographic assessment of treatment response, and was a included to assess the value of early MRI in disease monitoring.

Patients underwent conventional MRI as part of the CABARET trial protocol. These studies normally also included PWI. The majority of investigations were undertaken on a 3-Tesla scanner, with the remainder performed on 1.5-T machines. Although specific MRI protocols differed slightly between patients, they typically included T1 pre- and post-contrast administration, FLAIR, DWI and T2-weighted scans.

Dynamic susceptibility-weighted MRI was used for PWI whereby dynamic MR images were acquired during a bolus IV administration of contrast agent. A pragmatic attempt was made to acquire the data in accordance with previous recommendations [12] whilst keeping the total 
contrast agent dose within recommended limits, and obtaining acceptable slice coverage and image signal-to-noise ratio. To reduce the effect of contrast leakage on CBV calculations, a $5 \mathrm{~mL}$ bolus of gadolinium contrast agent (Magnevist ${ }^{\circledR}$, Bayer, Germany) was administered to the patient five minutes prior to the PWI acquisition at a rate of $1 \mathrm{~mL} / \mathrm{s}$ followed by a $15 \mathrm{~mL}$ saline flush. For PWI, dynamic gradient-echo EPI images were then acquired every 1.5 to 3 seconds. After approximately 10 to 15 seconds of scanning, a $10 \mathrm{~mL}$ bolus of contrast agent was administered at a rate of $5 \mathrm{~mL} / \mathrm{s}$ followed by a $30 \mathrm{~mL}$ saline flush. Due to the differences in MR system configurations the TR $(1.2-3 \mathrm{~s})$, TE $(30-60 \mathrm{~ms})$, in-plane resolution $(1.2-3 \mathrm{~mm})$, slice thickness $(4-7 \mathrm{~mm})$, slice number $(14-20)$ and total acquisition times $(70-100 \mathrm{~s})$ were scanner dependent.

\section{Post-Acquisition Imaging Analysis}

All imaging was transferred to an external computing station for post-acquisition processing. Using Analyze 10.0 (Biomedical Imaging Resource, Mayo Clinic, Rochester, MN), tumor regions of interest (ROIs) were created from contrast-enhancing regions on T1-weighted sequences (T1+C ROI) and hyperintense regions on FLAIR sequences (FLAIR ROI). Cystic cavities and necrotic cores were not included. A further ROI was created from T2-weighted normal-appearing white matter (NAWM) of the contralateral centrum semiovale.

Perfusion-weighted imaging data were converted to maps proportional to contrast agent concentration to correct for blood vessel leakage in Matlab ${ }^{\circledR} 7.11$ (Mathworks, Inc., MA, USA), and maps proportional to $\mathrm{CBV}$ were then created by numerical integration on a voxel by voxel basis [12]. The tumor and NAWM ROIs were then co-registered and re-sampled onto the CBV 
map frame of reference via a nine-parameter affine co-registration (MINC tools, Montreal Neurological Institute) of the T1 post-contrast, FLAIR and T2 scans to the first phase of the PWI images. Relative CBV (rCBV) maps were subsequently created, relative to the NAWM ROI, again using Matlab ${ }^{\circledR}$. Tumor voxel rCBV histograms were then created for the T1+C and FLAIR ROIs.

Interval and Serial Imaging Characteristics

For each patient, T1 contrast-enhancing and FLAIR-hyperintense tumor volumes $\left(\mathrm{cm}^{3}\right)$ were calculated for the three (pre-treatment, and 4- and 8-week follow-up) imaging time-points by integrating the number of voxels within each corresponding ROI by the voxel volumes within Matlab $^{\circledR}$. Traditional tumor rCBV histogram statistics (mean, median, mode and maximum) were obtained from the histogram data of each tumor ROI. Additionally, a novel rCBV measurement was calculated to give a volumetric indicator of total tumor perfusion load. This was achieved by determining the numerical integral of the voxel rCBV histogram for a given tumor ROI $\left(\mathrm{rCBV}_{\text {load }}\right)$.

Values of traditional tumor rCBV histogram statistics, and $\mathrm{rCBV}_{\text {load }}$, for each imaging time-point were then compared to determine serial changes in these characteristics, expressed as a percentage. Definitions of volumetric progression (> $40 \%$ increase in volume) and response (> $65 \%$ reduction in volume) were applied to changes in $\mathrm{T} 1+\mathrm{C}$ and FLAIR tumor volumes, as well as the volumetric perfusion measurement $\mathrm{rCBV}_{\text {load }}$, to determine rates of radiographic progression and response $[13,14]$. 


\section{Statistical Analysis}

Statistical analysis was performed using Prism 5 for Mac OS X (GraphPad Software, Inc., San Diego). Correlations between rCBV measures and outcome were investigated using the Spearman correlation test. Patients surviving at time of analysis were censored at that date. Patients were also dichotomized to low and high $\mathrm{rCBV}_{\text {mean }}, \mathrm{rCBV}_{\text {median }}, \mathrm{rCBV}_{\text {mode }}, \mathrm{rCBV}_{\text {max }}$, and $\mathrm{rCBV}_{\text {load }}$ groups based on the median value of each of the five parameters, at each imaging timepoint Log-rank tests were then used to compare the Kaplan-Meier survival curves of these groups. The same analyses were applied to the percentage change in rCBV values at each followup imaging time-point. Statistical significance was considered to be $\mathrm{p}<0.05$ for all tests.

\section{Results}

Patient Demographics and Outcome

A total of 122 patients were enrolled in the CABARET trial, including 15 from our institutions between April 2011 and Feb 2012. For these 15 patients, the mean patient age was 49 years, with more males recruited $(n=9)$. Tumors were most commonly located in the frontal lobe $(n=5)$. The majority of patients $(n=12)$ exhibited good functional status at enrolment (KPS score 80 - 100), with three patients displaying moderate functional status (KPS score 50 $70)$.

At the time of analysis only one patient was alive and was censored for outcome analyses. One patient underwent further resection prior to commencement of treatment to confirm recurrence rather than radiation necrosis. This patient was excluded from response analyses as any change in tumor volume would be the result of treatment plus surgery. One 
patient discontinued treatment after four months due to acute renal impairment potentially caused by bevacizumab. Outcome data from this patient has been included. Progression-free and overall survival data was available for all patients. Median PFS and OS was 23 weeks (standard deviation (SD) \pm 24 weeks) and 45 weeks ( $\mathrm{SD} \pm 22$ weeks) respectively.

\section{Pre-Treatment MRI}

All patients had conventional pre-treatment MRI available for analysis. Nine out of 15 MR scans were performed on the same 3T machine. Scans were undertaken on average 24 days ( $\mathrm{SD} \pm 23$ days) prior to commencing treatment. The average contrast-enhancing tumor volume was $27 \mathrm{~cm}^{3}\left(\mathrm{SD} \pm 23 \mathrm{~cm}^{3}\right.$ ), while the average FLAIR hyperintense volume was $137 \mathrm{~cm}^{3}$ (SD \pm $\left.56 \mathrm{~cm}^{3}\right)$.

Pre-treatment MR imaging also included PWI for 13 patients. Based on Spearman analysis, no rCBV measure was found to significantly correlate with PFS or OS. However, Kaplan-Meier analysis demonstrated a significant difference in OS curves between patients with low versus high $\mathrm{rCBV}_{\text {median }}$ (Fig. 1a). Median survival was reduced by 18 weeks in patients with high rCBVs (53 vs. 35 weeks).

\section{4-week Follow-up MRI}

All patients had the initial follow-up MRI available for analysis, undertaken on average 26 days ( $\mathrm{SD} \pm 3$ days) following commencing treatment. 14 out of 15 4-week MR scans were performed on the same $3 \mathrm{~T}$ machine. The average contrast-enhancing tumor volume was $10 \mathrm{~cm}^{3}$ $\left(\mathrm{SD} \pm 10 \mathrm{~cm}^{3}\right)$, while the average FLAIR hyperintense volume was $70 \mathrm{~cm}^{3}\left(\mathrm{SD} \pm 27 \mathrm{~cm}^{3}\right)$. 
Spearman analysis of PWI revealed significant negative correlations between tumor rCBVs and both PFS and OS (Table 1). Kaplan-Meier analysis demonstrated a significant reduction in PFS in patients with large $\mathrm{rCBV}_{\text {load }}$ (median PFS 23 vs. 15 weeks; Fig. 1b). Significant reductions in OS were demonstrated in patients with high tumor rCBVs (Fig. 1c and 1d). High $\mathrm{rCBV}_{\text {median }}$ and $\mathrm{rCBV}_{\max }$ decreased median $\mathrm{OS}$ by 14 (52 vs. 38) and 21 (52 vs. 31) weeks respectively.

8-week Follow-up MRI

One patient had clinical and radiographic evidence of disease progression after 4-weeks follow-up and was excluded from analysis of the 8-week follow-up MRI. The remaining 14 patients all had MRI (including PWI) available, which was undertaken on average 55 days (SD \pm 7 days) following commencing treatment, and 13/14 were perfomed on the 3T machine. The average contrast-enhancing tumor volume was $6 \mathrm{~cm}^{3}\left(\mathrm{SD} \pm 9 \mathrm{~cm}^{3}\right)$, while the average FLAIR hyperintense volume was $60 \mathrm{~cm}^{3}\left(\mathrm{SD} \pm 27 \mathrm{~cm}^{3}\right)$.

Spearman analysis of PWI revealed significant negative correlations between tumor rCBVs and both PFS and OS (Table 1). Kaplan-Meier analysis demonstrated a significant reduction in PFS in patients with large $\mathrm{rCBV}_{\text {load }}$ (median PFS 32 vs. 16 weeks; Fig. 2a).

Significant reductions in OS were also demonstrated in patients with high tumor rCBVs based on T1+C ROIs (Fig. 2b, c and d). Median OS was reduced by 13 (54 vs. 41) weeks in patients with high $\mathrm{rCBV}_{\text {mean }}$, and by 16 (54 vs. 38) weeks in patients with high $\mathrm{rCBV}_{\text {mode }}$ and large $\mathrm{rCBV}_{\text {load }}$ (Fig. 2b, c and d). 
Predicting response to Bevacizumab based on Volumetric Criteria and PWI

Comparing pre-treatment to follow-up MRIs gave volumetric response rates of $50 \%$ at four weeks and $69 \%$ at eight weeks using conventional T1 post-contrast imaging. FLAIR imaging gave lower response rates (14 and 35\%), whilst including changes in perfusion through $\mathrm{rCBV}_{\text {load }}$ returned higher response rates (45 and 91\%). Volumetric progression was demonstrated only on conventional $\mathrm{T} 1$ post-contrast imaging for one patient, giving a rate of progression of $7 \%$.

Comparisons between outcome and changes in rCBV at 8 weeks, based on T1+C ROIs are shown in Fig. 3 and Table 2. Spearman and Kaplan Meier analyses both demonstrated that patients with the greatest reductions in rCBV had improved PFS and OS. Median OS was increased by 30 weeks in patients with the greatest reduction in $\mathrm{rCBV}_{\text {load }}$ at 8 weeks compared to pre-treatment imaging (Fig. 3b). No significant correlations were seen at the 4-week MRI or using changes in FLAIR ROI rCBVs.

\section{Discussion}

Imaging-based assessment of response to therapy in GBM has traditionally involved twodimensional measurements of contrast-enhancing tumor regions as recommended by MacDonald and colleagues in 1990 [15]. Limitations with this criteria became increasingly apparent as therapy-induced pseudo-progression and pseudo-response gained recognition. This prompted an updated response criteria (RANO) to be published in 2010, including non-enhancing regions of tumor in the imaging-based assessment of response [3]. As volumetric analysis and advanced 
imaging techniques such as PWI become more accessible it is likely that they will become incorporated into future revisions [14].

The current study investigated the ability of PWI to predict outcome in patients with rGBM treated with bevacizumab. Pre-treatment as well as early follow-up imaging were examined for associations with PFS and OS. Changes in perfusion imaging over time were also analyzed for links with outcome.

Fifteen patients were recruited for the CABARET trial from our institutions, and all were included in this analysis. Follow-up for all patients was complete and median PFS and OS were 23 and 45 weeks respectively. Compared with historical data, this cohort did far better than expected for patients with rGBM (PFS $\sim 10$ weeks; OS 30 weeks [16]). However, their outcomes are comparable to results from other phase II trials of bevacizumab therapy in rGBM patients [17-21].

In this study, patients with higher median rCBV within FLAIR hyperintense tumor volumes on pre-treatment MRI were found to have significantly reduced OS compared to those with lower values (53 vs. 35 week; Fig. 1a). This may simply reflect the poorer prognosis seen in patients with more vascular tumors, though it also suggests that these patients may be less likely to benefit from anti-angiogenic therapies such as bevacizumab. Perfusion MRI at the time of GBM recurrence may therefore be useful in selecting which patients should be considered for anti-angiogenic therapy.

Following the commencement of anti-angiogenic agents, PWI analysis of the contrastenhancing tumor regions, rather than FLAIR hyperintense regions, appears to become more important in monitoring response and predicting outcome. After four weeks of therapy with 
bevacizumab, median rCBV on FLAIR ROIs correlated with both PFS and OS, but stronger and more significant correlations with OS were seen with $\mathrm{T} 1+\mathrm{C}$ ROI rCBV histogram statistics. By eight weeks of therapy, all correlations were lost with FLAIR ROI rCBV histogram statistics, but became stronger with $\mathrm{T} 1+\mathrm{C}$ ROIs. The reason for this shift in significance from FLAIR to $\mathrm{T} 1+\mathrm{C}$ during treatment is uncertain, but may relate to a greater sensitivity of contrast-enhancing tumor to the effects of anti-angiogenic agents. Changes in tumor volume with treatment were also greatest on T1 post-contrast imaging.

Follow-up PWI as early as four weeks was able to predict outcome in patients receiving bevacizumab. At this imaging time point, patients with high rCBVs demonstrated reductions in OS between 14 and 21 weeks compared to patients with low values. This predictive value persisted and strengthened by the 8-week follow-up MRI. Again, these findings may reflect the poorer prognosis associated with more highly vascular tumors, but it is interesting to note that also patients with the smallest reductions, or any increase, in tumor rCBV measures following commencement of treatment were demonstrated to have worse PFS and OS.

The strongest and most significant correlations between these PWI changes with bevacizumab therapy and outcome were seen with $\mathrm{rCBV}_{\text {load }}$ This novel $\mathrm{rCBV}$ measurement was developed to estimate the total volume of blood vessels within a given tumor. Unlike histogram statistics, which do not vary with tumor size, $\mathrm{rCBV}_{\text {load }}$ takes into account the entire mass of abnormal tumor vasculature that is responding to treatment. Increases in $\mathrm{rCBV}_{\text {load }}$ at the 4- and 8week follow-up MRIs were associated with both poorer PFS and OS.

Imaging response rates were also assessed using true volumetric analysis. Using RECIST criteria, response rates of $50 \%$ and $69 \%$ were seen at 4- and 8-week follow-up respectively. Response rates from other phase II trials of bevacizumab for rGBM using MacDonald imaging 
criteria range from $23-57 \%$ following long-term follow-up [10, 17-21]. The increased response rate seen in the current study may relate to a number of factors including differences in treatment regimens and patient populations. However, one phase II study of bevacizumab in rGBM patients has directly compared MacDonald criteria to volumetric analysis of imaging response at early timepoints (3 and 21 days), and also reported higher response rates with the volumetric analysis [8]. This suggests that volumetric analysis may be a more sensitive way of monitoring GBM response to therapy.

A shortcoming of this study was that nine of 15 subjects had baseline scans on a $1.5 \mathrm{~T}$ scanner and underwent further MRI studies on a 3T scanner. This was a result of all baseline scans for this clinical trial being taken from the subjects standard clinical MRI exams which were spread over our institution's three MRI scanners and some external Radiology sites. For the perfusion scanning the main effect of this would have been lower image resolution and thicker slices at $1.5 \mathrm{~T}$. The effect on $\mathrm{rCBV}$ measures would have been minimal as the main difference would have been the differing T1 contamination of the rCBV values. This was carefully corrected for as previously recommended. In a recent study, 1.5 and $3 \mathrm{~T} \mathrm{rCBV}$ values were found to be highly correlated (ICC $=0.92$ [95\% CI, 0.85-0.97]) [22].

Although the CABARET trial is a large, well designed, multi-center, prospective clinical trial, this report represents an essentially retrospective ad hoc analysis of the data obtained from one of its recruiting centers. This leads to a number of inherent limitations including small sample size. The lack of PWI in the CABARET protocol also meant that not all patients in the current study underwent pre-treatment PWI (13 of 15). Despite these problems, highly significant correlations and differences in outcome were still observable. 


\section{Conclusion}

Findings from this study suggest that PWI may be of significant clinical utility in managing patients with rGBM treated with anti-angiogenic agents such as bevacizumab. We have demonstrated that pre-treatment PWI may be able to help in selecting which patients are likely to benefit most from bevacizumab. We have also shown that early follow-up imaging, even at 4 weeks, may help in identifying which patients are less likely to gain from continued use of anti-angiogenic agents and for whom other treatments should be sought. Monitoring changes in the entire tumor blood vessel volume, with $\mathrm{rCBV}_{\text {load, }}$ appears to be the most useful way to identify such patients. These findings will necessarily need to be confirmed in larger studies. 


\section{Figure legends}

Fig. 1 Kaplan-Meier analyses of pre-treatment $(\boldsymbol{A})$ and 4-week follow-up (B, C, and $\boldsymbol{D})$ PWI with respect to survival. A. Significantly reduced overall survival is demonstrated in patients with high rCBV $_{\text {median }}$ on pre-treatment PWI B. Significantly reduced progressionfree survival is demonstrated in patients with high rCBV load on 4-week follow-up PWI. C and D. Overall survival is significantly reduced in patients with high $r C B V_{\text {median }}$ and high $r C B V_{\max }$ on 4-week follow-up PWI. $r C B V=$ relative cerebral blood volume; $P W I=$ perfusion-weighted imaging

Fig. 2 Kaplan-Meier analyses of 8-week follow-up PWI with respect to survival. A. Significantly reduced progression-free survival is demonstrated in patients with high $\mathrm{rCBV}_{\text {load }}$ B, $\boldsymbol{C}$ and $\boldsymbol{D}$. Overall survival is significantly reduced in patients with high $r C B V_{\text {mean }}$, high $r C B V_{\text {mode }}$ and high $r C B V_{\text {load }} r C B V=$ relative cerebral blood volume; $P W I=$ perfusion-weighted imaging

Fig. 3 Kaplan-Meier analyses of changes in PWI at 8-week follow-up MRI with respect to survival. 8-week PWI is compared with both pre-treatment (A and $\boldsymbol{B})$ and 4-week follow-up (C and $\boldsymbol{D})$ PWI. All rCBVs are based on contrast-enhancing tumor volumes $(T 1+C$ ROIs) 


\section{References}

1. Cohen MH, Shen YL, Keegan P, Pazdur R (2009) FDA drug approval summary: bevacizumab (Avastin) as treatment of recurrent glioblastoma multiforme. Oncologist 14:1131-1138. doi: 10.1634/theoncologist.2009-0121

2. Khasraw M, Simeonovic M, Grommes C (2012) Bevacizumab for the treatment of highgrade glioma. Expert Opin Biol Ther 12:1101-1111. doi: 10.1517/14712598.2012.694422

3. Wen PY, Macdonald DR, Reardon DA, et al (2010) Updated response assessment criteria for high-grade gliomas: response assessment in neuro-oncology working group. Journal of Clinical Oncology 28:1963-1972. doi: 10.1200/JCO.2009.26.3541

4. Rüegg C, Meuwly J-Y, Driscoll R, et al (2003) The quest for surrogate markers of angiogenesis: a paradigm for translational research in tumor angiogenesis and antiangiogenesis trials. Curr Mol Med 3:673-691.

5. Gossmann A, Helbich TH, Kuriyama N, et al (2002) Dynamic contrast-enhanced magnetic resonance imaging as a surrogate marker of tumor response to anti-angiogenic therapy in a xenograft model of glioblastoma multiforme. J Magn Reson Imaging 15:233240.

6. Keunen O, Johansson M, Oudin A, et al (2011) Anti-VEGF treatment reduces blood supply and increases tumor cell invasion in glioblastoma. Proceedings of the National Academy of Sciences 108:3749-3754. doi: 10.1073/pnas.1014480108

7. Pechman KR, Donohoe DL, Bedekar DP, et al (2011) Characterization of bevacizumab dose response relationship in U87 brain tumors using magnetic resonance imaging measures of enhancing tumor volume and relative cerebral blood volume. J Neurooncol 105:233-239. doi: 10.1007/s11060-011-0591-8

8. Verhoeff JJC, Lavini C, van Linde ME, et al (2010) Bevacizumab and dose-intense temozolomide in recurrent high-grade glioma. Ann Oncol 21:1723-1727. doi: 10.1093/annonc/mdp591

9. Sawlani RN, Raizer J, Horowitz SW, et al (2010) Glioblastoma: a method for predicting response to antiangiogenic chemotherapy by using MR perfusion imaging--pilot study. Radiology 255:622-628. doi: 10.1148/radiol.10091341

10. Kreisl TN, Zhang W, Odia Y, et al (2011) A phase II trial of single-agent bevacizumab in patients with recurrent anaplastic glioma. Neuro-oncology 13:1143-1150. doi: 10.1093/neuonc/nor091

11. Laviolette PS, Cohen AD, Prah MA, et al (2013) Vascular change measured with independent component analysis of dynamic susceptibility contrast MRI predicts bevacizumab response in high-grade glioma. Neuro-oncology 15:442-450. doi: 10.1093/neuonc/nos323 
12. Paulson ES, Schmainda KM (2008) Comparison of dynamic susceptibility-weighted contrast-enhanced MR methods: recommendations for measuring relative cerebral blood volume in brain tumors. Radiology 249:601-613. doi: 10.1148/radiol.2492071659

13. Therasse P, Arbuck SG, Eisenhauer EA, et al (2000) New guidelines to evaluate the response to treatment in solid tumors. European Organization for Research and Treatment of Cancer, National Cancer Institute of the United States, National Cancer Institute of Canada. J Natl Cancer Inst 92:205-216.

14. Galanis E, Buckner JC, Maurer MJ, et al (2006) Validation of neuroradiologic response assessment in gliomas: measurement by RECIST, two-dimensional, computer-assisted tumor area, and computer-assisted tumor volume methods. Neuro-oncology 8:156-165. doi: 10.1215/15228517-2005-005

15. Macdonald DR, Cascino TL, Schold SC, Cairncross JG (1990) Response criteria for phase II studies of supratentorial malignant glioma. J Clin Oncol 8:1277-1280.

16. Wong ET, Hess KR, Gleason MJ, et al (1999) Outcomes and prognostic factors in recurrent glioma patients enrolled onto phase II clinical trials. J Clin Oncol 17:2572-2578.

17. Vredenburgh JJ, Desjardins A, Herndon JE, et al (2007) Bevacizumab plus irinotecan in recurrent glioblastoma multiforme. Journal of Clinical Oncology 25:4722-4729. doi: 10.1200/JCO.2007.12.2440

18. Reardon DA, Desjardins A, Vredenburgh JJ, et al (2009) Metronomic chemotherapy with daily, oral etoposide plus bevacizumab for recurrent malignant glioma: a phase II study. Br J Cancer 101:1986-1994. doi: 10.1038/sj.bjc.6605412

19. Sathornsumetee S, Desjardins A, Vredenburgh JJ, et al (2010) Phase II trial of bevacizumab and erlotinib in patients with recurrent malignant glioma. Neuro-oncology 12:1300-1310. doi: 10.1093/neuonc/noq099

20. Møller S, Grunnet K, Hansen S, et al (2012) A phase II trial with bevacizumab and irinotecan for patients with primary brain tumors and progression after standard therapy. Acta Oncol 51:797-804. doi: 10.3109/0284186X.2012.681063

21. Nagane M, Nishikawa R, Narita Y, et al (2012) Phase II study of single-agent bevacizumab in Japanese patients with recurrent malignant glioma. Jpn J Clin Oncol 42:887-895. doi: 10.1093/jjco/hys121

22. Tselikas L, Souillard-Scemama R, Naggara O, et al (2015) Imaging of gliomas at 1.5 and 3 Tesla - A comparative study. Neuro-oncology 17:895-900. doi: 10.1093/neuonc/nou332 
A Overall survival vs. rCBV median on FLAIR ROI

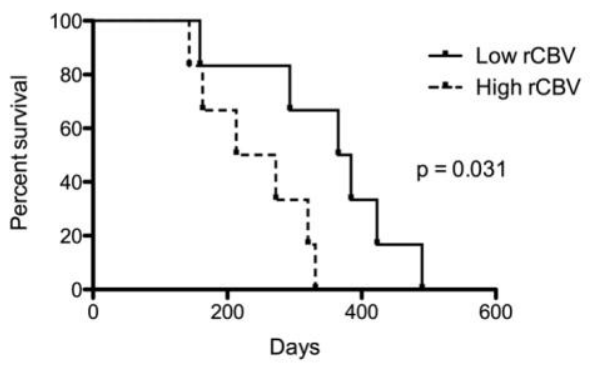

C Overall survival vs. $\mathrm{rCBV}$ median on $\mathrm{T} 1+\mathrm{C} \mathrm{ROI}$

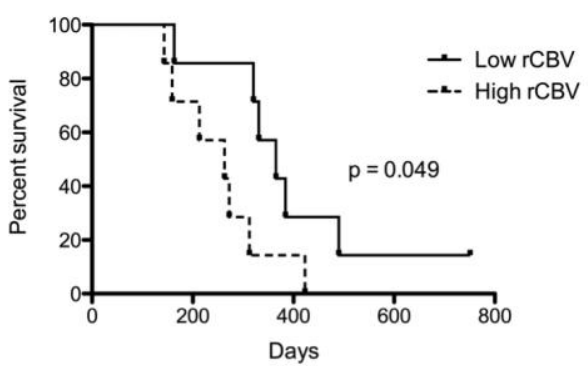

B Progression-free survival vs. rCBV load on FLAIR ROI

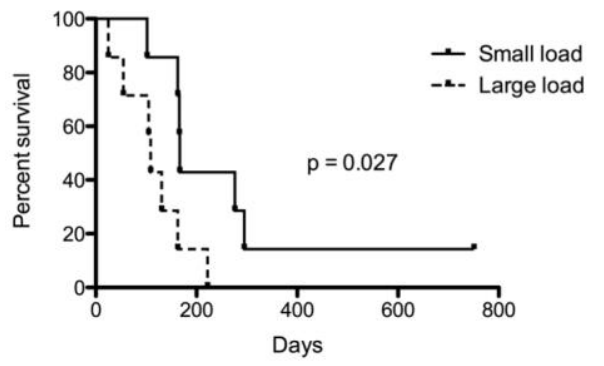

D Overall survival vs. rCBV maximum on $T 1+C \mathrm{ROI}$

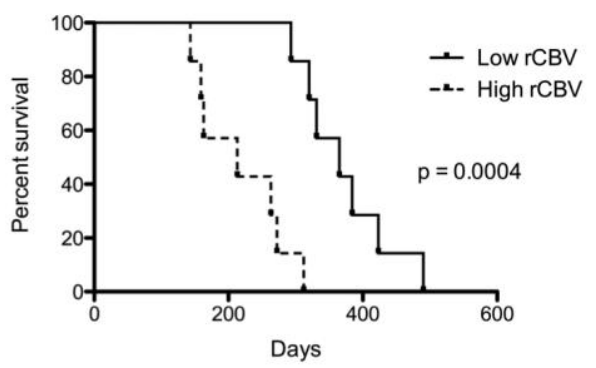


A Progression-free survival vs. rCBV load on T1+C ROI

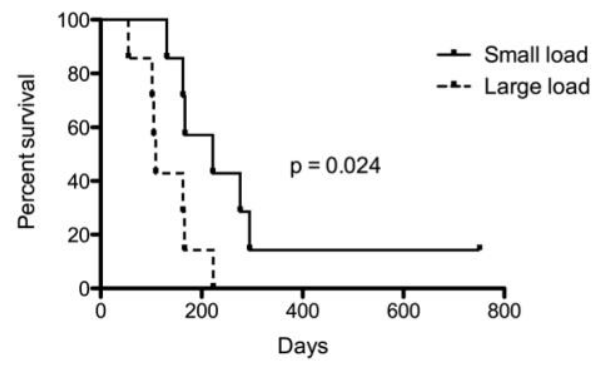

C

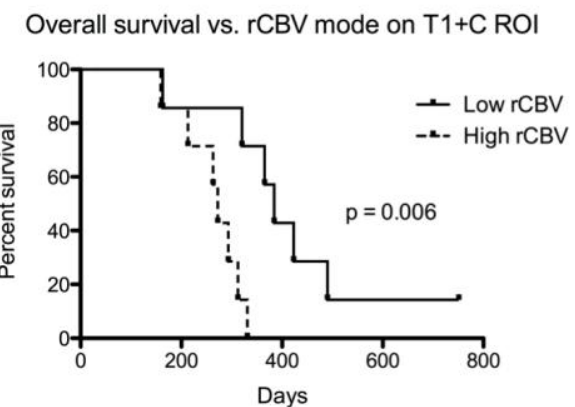

B

Overall survival vs. rCBV mean on $\mathrm{T} 1+\mathrm{C} \mathrm{ROI}$

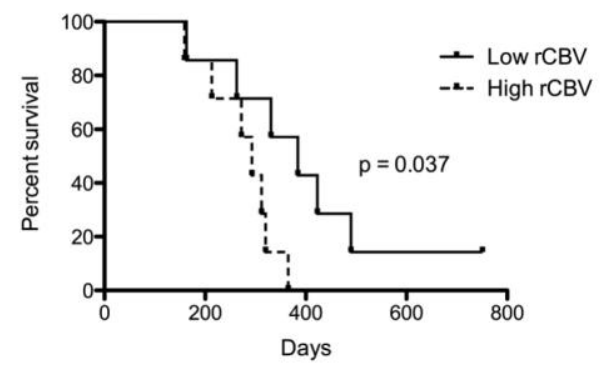

D

Overall survival vs. rCBV load on $T 1+C$ ROI

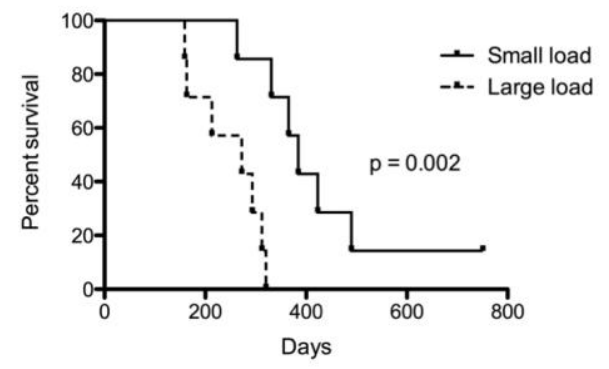


A Progression-free survival vs. change in rCBV load from pre-treatment PWI

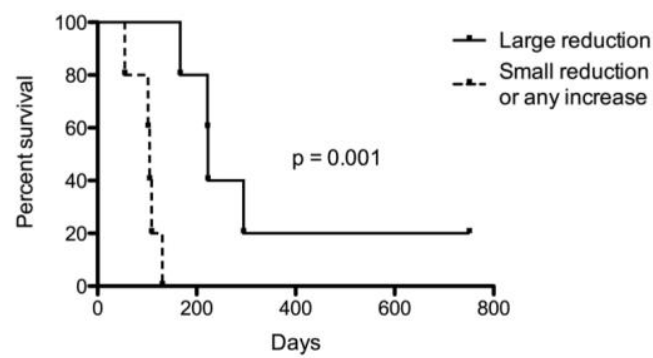

C

Progression-free survival vs. change in rCBV load from 4-week PWI

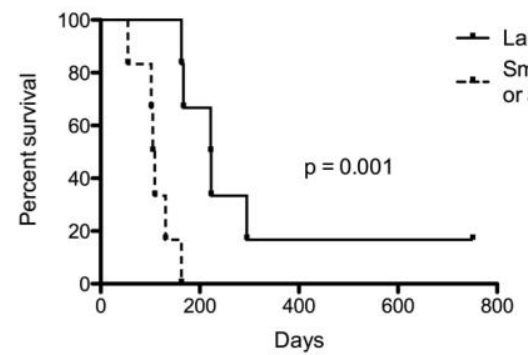

B Overall survival vs. change in rCBV load from pre-treatment $\mathrm{PWI}$

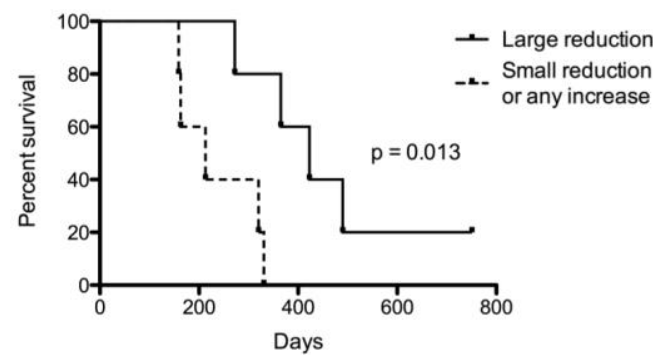

Overall survival vs. change in rCBV load from 4-week PWI

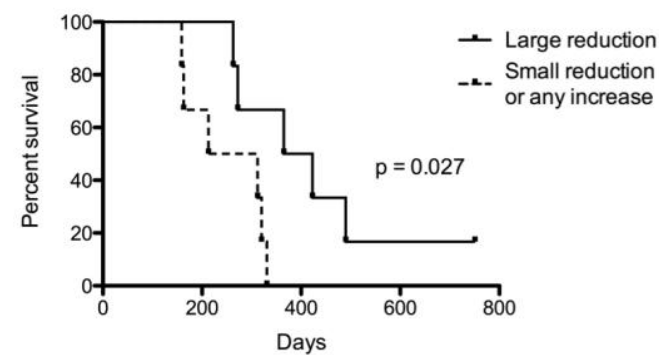

Tables

Table 1. Correlation of follow-up perfusion MRI with outcome

\begin{tabular}{|c|c|c|c|c|c|c|c|c|}
\hline & \multicolumn{4}{|c|}{ 4-week MRI } & \multicolumn{4}{|c|}{ 8-week MRI } \\
\hline & \multicolumn{2}{|c|}{ vs. PFS } & \multicolumn{2}{|c|}{ vs. OS } & \multicolumn{2}{|c|}{ vs. PFS } & \multicolumn{2}{|c|}{ vs. OS } \\
\hline & r value $^{\mathrm{a}}$ & $\mathrm{p}$ value & r value $^{a}$ & $\mathrm{p}$ value & r value $^{\mathrm{b}}$ & $\mathrm{p}$ value & rvalue $^{b}$ & $\mathrm{p}$ value \\
\hline \multicolumn{9}{|c|}{ T1+C ROI histogram statistics: } \\
\hline rCBV mean & -0.197 & 0.483 & -0.500 & 0.058 & -0.517 & 0.058 & -0.578 & 0.030 \\
\hline rCBV median & -0.166 & 0.555 & -0.433 & 0.107 & -0.634 & 0.015 & -0.719 & 0.004 \\
\hline rCBV mode & -0.365 & 0.182 & -0.561 & $\mathbf{0 . 0 3 0}$ & -0.466 & 0.093 & -0.596 & 0.025 \\
\hline rCBV maximum & -0.420 & 0.119 & -0.643 & 0.010 & -0.416 & 0.139 & -0.451 & 0.106 \\
\hline rCBV load & -0.456 & 0.089 & -0.532 & 0.041 & -0.700 & 0.005 & -0.649 & 0.012 \\
\hline \multicolumn{9}{|c|}{ FLAIR ROI histogram statistics: } \\
\hline rCBV mean & -0.408 & 0.132 & -0.443 & 0.098 & 0.018 & 0.952 & -0.293 & 0.310 \\
\hline rCBV median & -0.523 & 0.045 & -0.532 & 0.041 & 0.152 & 0.603 & -0.170 & 0.562 \\
\hline rCBV mode & -0.061 & 0.830 & -0.218 & 0.435 & 0.029 & 0.923 & -0.326 & 0.256 \\
\hline
\end{tabular}




\begin{tabular}{lllll|llll} 
rCBV maximum & -0.477 & 0.072 & -0.486 & 0.066 & -0.079 & 0.788 & -0.433 & 0.122 \\
rCBV load & $\mathbf{- 0 . 5 5 8}$ & $\mathbf{0 . 0 3 1}$ & -0.364 & 0.182 & $\mathbf{- 0 . 5 5 9}$ & $\mathbf{0 . 0 3 8}$ & -0.499 & 0.069 \\
& & & & & & & & \\
\hline
\end{tabular}

Abbreviations: rCBV, relative cerebral blood volume; T1+C ROI, contrast-enhancing tumor; FLAIR ROI, hyperintense tumor.

${ }^{\mathrm{a}}$ Spearman correlation test, degrees of freedom $=14 ;{ }^{\mathrm{b}}$ Spearman correlation test, degrees of freedom $=13$.

Table 2. Correlation of changes in $\mathrm{PWI}^{\mathrm{a}}$ at 8 weeks with outcome

\begin{tabular}{|c|c|c|c|c|}
\hline & \multicolumn{2}{|c|}{ vs. progression-free survival } & \multicolumn{2}{|c|}{ vs. overall survival } \\
\hline & r value & $\mathrm{p}$ value & $\mathrm{r}$ value & $\mathrm{p}$ value \\
\hline \multicolumn{5}{|c|}{ Compared to pre-treatment MRI: } \\
\hline rCBV mean & -0.501 & 0.122 & -0.447 & 0.173 \\
\hline rCBV median & -0.646 & 0.037 & -0.500 & 0.122 \\
\hline rCBV mode & -0.182 & 0.595 & -0.182 & 0.595 \\
\hline rCBV maximum & -0.555 & 0.082 & -0.464 & 0.155 \\
\hline rCBV load & -0.898 & 0.0004 & -0.770 & 0.007 \\
\hline \multicolumn{5}{|c|}{ Compared to 4-week follow-up MRI: } \\
\hline rCBV mean & -0.639 & 0.019 & -0.402 & 0.174 \\
\hline rCBV median & -0.671 & 0.012 & -0.434 & 0.138 \\
\hline rCBV mode & -0.430 & 0.143 & -0.451 & 0.122 \\
\hline rCBV maximum & -0.278 & 0.358 & -0.038 & 0.901 \\
\hline rCBV load & -0.801 & 0.001 & -0.614 & 0.026 \\
\hline
\end{tabular}

Abbreviations: MRI, magnetic resonance imaging; PWI, perfusion-weighted imaging; rCBV, relative cerebral blood volume.

${ }^{\text {a }}$ Based on contrast-enhancing tumor volumes.

\section{Supplementary Material 1. CABARET Inclusion and Exclusion Criteria.}

\section{Key Inclusion Criteria}

For inclusion in this study, all of the following inclusion criteria must be fulfilled:

1. Patients with glioblastoma multiforme (GBM) with a tissue diagnosis that has been established following either a surgical resection or biopsy, and who have had prior treatment with both radiotherapy and temozolomide (alone or in combination) 
2. Recurrent/progressive disease confirmed by surgical resection or MRI (measurable disease according to RANO criteria). Measurable disease will be characterized by all of the following:

a. at least one site of bi-dimensionally measurable disease

b. two perpendicular diameters of at least $10 \mathrm{~mm}$, visible on 2 or more axial slices that are preferably, at most, $5 \mathrm{~mm}$ thick with 0-mm skip (or at least two times the slice thickness if the MRI is performed with thicker slices)

c. must be measured using contrast-enhanced MRI

d. MRI showing progression must be performed within 14 days before randomization and at least 12 weeks post cessation of radiotherapy or stereotactic radiosurgery. The MRI must be compared with a prior MRI performed postradiotherapy

3. Craniotomy or intracranial biopsy site must be adequately healed; free of drainage or cellulitis, and the underlying cranioplasty must appear intact at the time of randomization. Study treatment should be initiated greater than or equal to 28 days following the last surgical procedure (including biopsy, surgical resection, wound revision, or any other major surgery involving entry into a body cavity)

4. World Health Organization (WHO)/ Eastern Cooperative Oncology Group (ECOG) performance status less than or equal to 2

5. At least 3 (three) months must have elapsed since the cessation of radiotherapy

6. Adequate renal function (within 2 weeks prior to randomization):

a. Creatinine less than or equal to $1.25 \mathrm{x}$ upper limit of normal (ULN) or creatinine clearance rate $>60 \mathrm{~mL} / \mathrm{min}$ AND 
b. Urine dipstick for proteinuria $<2+$. Patients discovered to have greater than or equal to $2+$ proteinuria on dipstick urinalysis at baseline should undergo a 24 hour urine collection or urine protein/creatinine ratio and must demonstrate less than or equal to $1.0 \mathrm{~g}$ of protein in 24 hours

OR

c. Urine protein/creatinine ratio (UPC) less than or equal to 1.0.

7. Laboratory values (within 2 weeks prior to randomization):

a. Absolute neutrophil count (ANC) greater than or equal to $1.5 \times 10^{9} / \mathrm{L}$.

b. Leucocyte count $>3.0 \times 10^{9} / \mathrm{L}$

c. Platelets greater than or equal to $100 \times 10^{9} / \mathrm{L}$.

d. Hemoglobin greater than or equal to $100 \mathrm{~g} / \mathrm{L}$.

e. Total bilirubin less than or equal to $1.5 \mathrm{x}$ the ULN.

f. Aspartate aminotransferase (AST) and alanine aminotransferase (ALT) less than or equal to $2.5 \mathrm{x}$ ULN (less than or equal to $5 \mathrm{x}$ ULN when attributable to anticonvulsants)

g. Alkaline phosphatase less than or equal to $2.5 \mathrm{x}$ ULN.

8. International normalized ratio (INR) or prothrombin time (PT) (sec) and activated partial thromboplastin time (aPTT)

a. $1.5 \times$ ULN (except for subjects receiving anticoagulation therapy at the time of screening) in the absence of therapeutic intent to anticoagulate the subject.

b. Within therapeutic limits (according to the medical standard in the institution) in the presence of therapeutic intent to anticoagulate the subject at the time of screening. 
NOTE: As per American Society of Clinical Oncology (ASCO) guidelines, low molecular weight heparin (LMWH) is the preferred approach. Patients on warfarin at the time of screening must be switched to LMWH at least 2 weeks prior to randomization and commencement of study drug.

9. Signed informed consent

10. Age
a. Minimum: 18 years
b. Maximum: none

11. Gender

a. Both male and female

Key Exclusion Criteria

Patients are not eligible for this study if they fulfill one or more of the following exclusion criteria:

1. Prior therapy with bevacizumab or any other vascular endothelial growth factor (VEGF) inhibitor/VEGF receptor tyrosine kinase inhibitor or epidermal growth factor receptor (EGFR) inhibitor

2. Treatment with concomitant investigational drug or has received an investigational agent within the past 30 days prior to the first dose of bevacizumab +/- carboplatin

3. Known hypersensitivity to any excipients of bevacizumab formulation or to carboplatin.

4. Hypersensitivity to Chinese hamster ovary cell products or other recombinant human or humanized antibody 
5. The subject is receiving warfarin (or other coumarin derivatives) and is unable to switch to LMWH 14 days prior to randomization

6. Have had any surgery, open biopsy, intracranial biopsy, ventriculoperitoneal shunt or significant traumatic injury within 4 weeks prior to start of treatment on this study or who have not recovered from side effects of such therapy

7. Core biopsy (excluding intracranial biopsy) or other minor surgical procedure within 7 days prior to randomization. Placement of a central vascular access device (CVAD) if performed within 2 days prior to bevacizumab administration

8. Pregnancy or lactation

9. Patient (male or female) is not willing to use highly effective methods of contraception (e.g. double barrier method) during treatment and for 6 months (male or female) after the end of treatment

10. Evidence of recent hemorrhage on MRI of the brain. However patients with clinically asymptomatic presence of hemosiderin, resolving hemorrhagic changes related to surgery, and presence of punctate hemorrhage in the tumor are permitted entry into the study

11. Calculated creatinine clearance (Cockroft-Gault) $<60 \mathrm{~mL} / \mathrm{min}$

12. Inability to undergo MRI (e.g. has a pacemaker)

13. Presence of any psychological, familial, sociological or geographical condition potentially hampering compliance with the study protocol and follow-up schedule, including alcohol dependence or drug abuse

14. Any of the following conditions: 
a. Inadequately controlled hypertension (defined as systolic blood pressure $>150$ $\mathrm{mmHg}$ and/or diastolic blood pressure $>100 \mathrm{mmHg}$ ); or prior history of hypertensive crisis or hypertensive encephalopathy

b. New York Heart Association (NYHA) Grade II or greater congestive heart failure

c. History of myocardial infarction, unstable angina, stroke or transient ischemic attack (TIA), or significant vascular disease (e.g. aortic aneurysm requiring surgical repair or recent peripheral arterial thrombosis) within 6 months prior to randomization

d. History of greater than or equal to grade 2 hemoptysis according to the National Cancer Institute Common Terminology Criteria for Adverse Events (NCICTCAE) version 4.0 criteria within 1 month prior to randomization

e. History of abdominal fistula, gastrointestinal perforation, or intracranial abscess within 6 months prior to randomization

f. History of coagulation disorder associated with bleeding or recurrent thrombotic events

g. Prior or co-existent malignancy except non-melanomatous skin cancer, or malignancy treated and disease free for $>5$ years

h. Concurrent illness, including serious non-healing wound, active ulcer or untreated bone fracture, that may jeopardize the ability of the patient to receive the procedures outlined in this protocol with reasonable safety 